\title{
Fungal Esophagitis Presenting With Esophagitis Dissecans Superficialis: Cause or Concurrence? A Diagnostic Conundrum
}

\author{
Rashmee Patil $^{\mathrm{a}}$, Tagore Sunkara ${ }^{\mathrm{b}, \mathrm{d}}$, Mel A. Ona ${ }^{\mathrm{b}}$, Vinaya Gaduputi ${ }^{\mathrm{c}}$, Madhavi Reddy ${ }^{\mathrm{b}}$
}

\begin{abstract}
Esophagitis dissecans superficialis (EDS) is a rare desquamative disorder of the esophagus. It is characterized by sheets of sloughed squamous tissue with normal underlying mucosa. We present a case of a 68-year-old man with severe anemia and a positive fecal occult blood test who was found to have features suggestive of EDS during endoscopy. However, histological features were suggestive of both EDS and fungal esophagitis. To our knowledge, this is the first reported case in literature which discusses the clinical scenario of fungal infection being a possible etiology rather than a commonly accepted differential diagnosis of EDS. We also review the clinical features, diagnostic criteria and treatment of EDS.
\end{abstract}

Keywords: Esophagitis dissecans superficialis; Sloughing esophagitis; Esophageal membrane

\section{Introduction}

Esophagitis dissecans superficialis (EDS) is a desquamative disorder of the esophagus that is characterized by sheets of sloughed squamous tissue with normal underlying mucosa. EDS is rare and fewer than 100 cases have been reported in the literature [1]. Many systemic diseases and medications have been associated with EDS; however, the exact etiology of EDS is unclear. We present a case of a 68-year-old male with history of alcohol abuse who presented to the emergency department

Manuscript accepted for publication December 15, 2016

aDepartment of Internal Medicine, Mount Sinai Health Systems, E 101st, New York, NY 10029, USA

bDepartment of Gastroenterology and Hepatology, The Brooklyn Hospital Center, Clinical Affiliate of The Mount Sinai Hospital, 121 Dekalb Ave., Brooklyn, NY 11201, USA

'Department of Gastroenterology and Hepatology, SBH Health System, 4422 Third Ave, Bronx, NY 10457, USA

${ }^{\mathrm{d} C}$ Corresponding Author: Tagore Sunkara, Department of Gastroenterology and Hepatology, The Brooklyn Hospital Center, Clinical Affiliate of The Mount Sinai Hospital, 121 Dekalb Avenue, Brooklyn, NY 11201, USA.

Email: tagoresunkara@hotmail.com

doi: https://doi.org/10.14740/gr739w
(ED) with severe anemia and was found to have typical findings of EDS on endoscopy.

\section{Case Report}

A 68-year-old male with history of alcohol abuse presented to the ED after syncope and fall. He denied abdominal pain, nausea, vomiting, difficulty swallowing, blood in the stool, change in bowel habits, or weight loss. He denied family history of colon cancer. Physical examination findings were unremarkable. Gastroenterology was consulted for evaluation of severe anemia; hemoglobin was $5.1 \mathrm{~g} / \mathrm{dL}$ (baseline $10 \mathrm{~g} / \mathrm{dL}$ ) and fecal occult blood tested positive. Computed tomography (CT) scan of his abdomen was negative for retroperitoneal hematoma and revealed chronic pancreatitis and diffuse fatty infiltration of the liver. Patient was resuscitated with multiple packed red blood cell transfusions with a resulting hemoglobin of $10 \mathrm{~g} / \mathrm{dL}$.

Esophagogastroduodenoscopy (EGD) and colonoscopy were performed. EGD revealed multiple, large, linear peeling strips of superficial, white-colored sloughing in the entire esophagus, which appeared suggestive of EDS (Fig. 1). The mucosal sloughing was easily removed with biopsy forceps with no bleeding seen from the underlying normal-appearing esophageal mucosa. Multiple biopsies were taken. There was

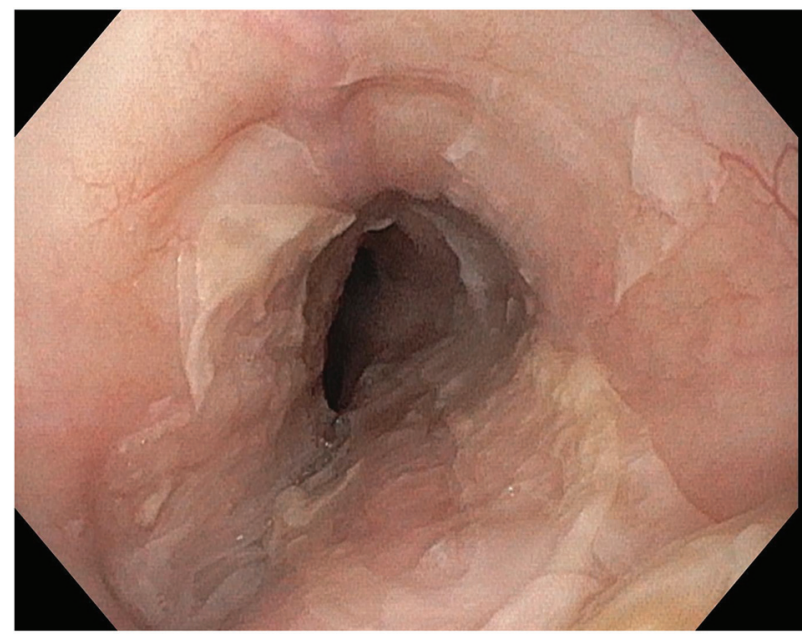

Figure 1. Esophagogastroduodenoscopy demonstrating sloughing of large fragments of the esophageal mucosa. 


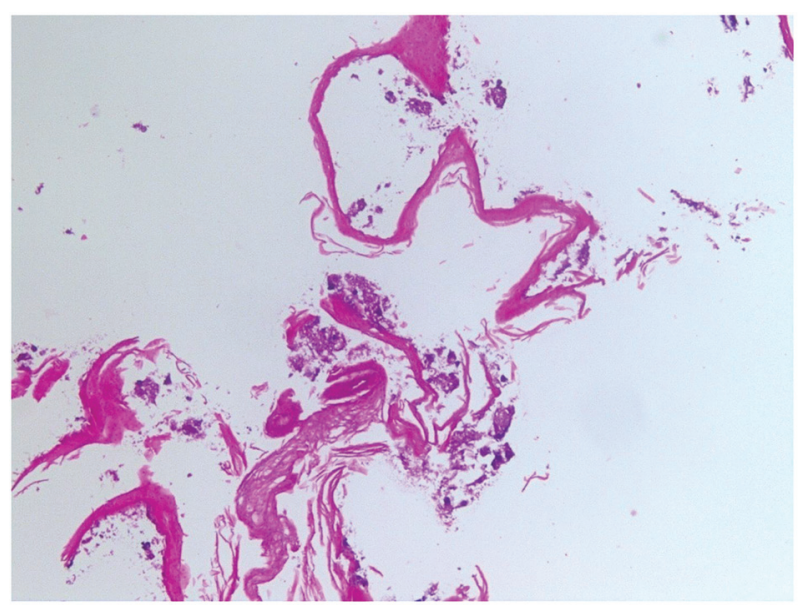

Figure 2. Superficial squamous mucosa on H\&E stain, with no basal layer seen (low power magnification).

mild erythema of the gastric body and antrum, while duodenum showed non-bleeding erosions. Colonoscopy revealed non-bleeding diverticulosis and two colon polyps, which were removed. The patient subsequently had an uneventful clinical course and was eventually discharged to a short-term rehabilitation facility with a course of anti-fungal therapy.

The esophageal biopsies consisted of detached squamous mucosa (Figs. 2 and 3) with fungal hyphae and bacteria, confirmed by periodic acid-Schiff (PAS) stain (Fig. 4). The histological findings fulfilled the diagnostic criteria of both EDS (mentioned below in discussion) and fungal esophagitis. This posed a hitherto unreported clinical scenario where fungal infection could be the etiology of EDS rather than a mere differential diagnosis.

\section{Discussion}

EDS is characterized by sloughing of large fragments of the esophageal mucosa and has been described as "sloughing es-

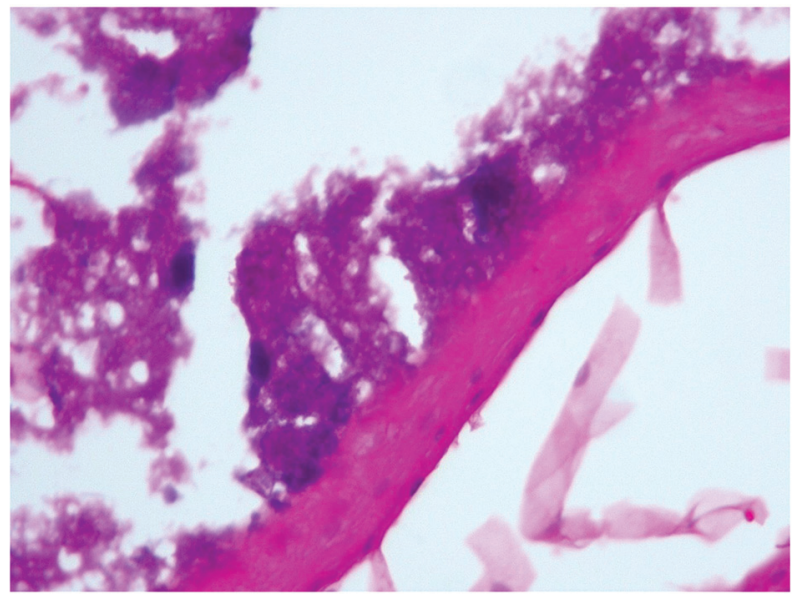

Figure 3. Superficial squamous mucosa on H\&E stain, with no basal layer seen (high power magnification).

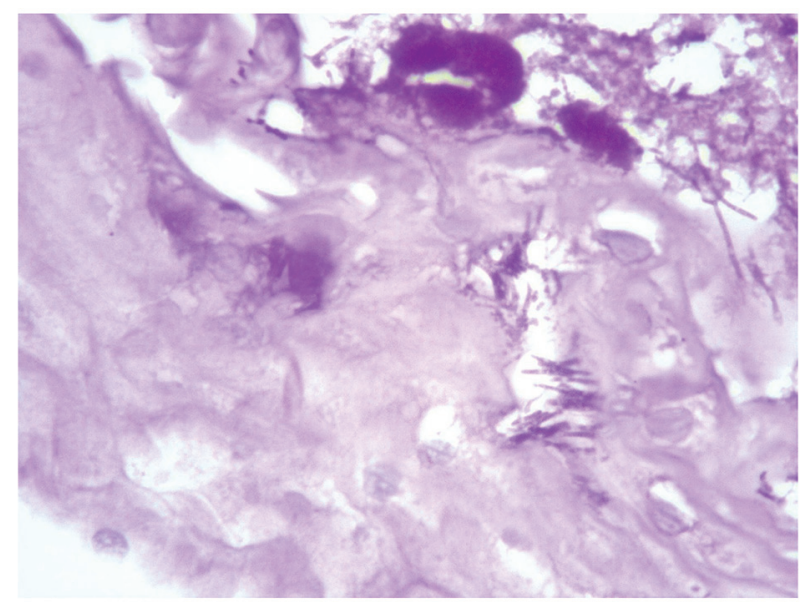

Figure 4. Periodic acid-Schiff stain showing fungal hyphae.

ophagitis" [2, 3]. EDS has been associated with medications such as bisphosphonates, non-steroidal anti-inflammatory drugs (NSAIDS), potassium chloride, and systemic diseases including celiac disease, collagen vascular disorders, and autoimmune bullous dermatoses. However, the direct mechanism of esophageal injury in EDS remains unclear. Pathogenesis of EDS might be a result of direct insult to the esophageal mucosa through physical, chemical, thermal, immunological mechanisms, or associated with a topical or ischemic injury $[4,5]$. EGD classically shows a stripped off mucosal appearance in the esophagus. Histology varies from sloughing off of squamous epithelium to parakeratosis to acute on chronic inflammation. EDS is a benign condition and endoscopy distinguishes it from other esophageal pathology.

\section{Clinical characteristics}

EDS symptoms include dysphagia, odynophagia, or symptoms of gastroesophageal reflux disease. Early reports of EDS in the literature have more dramatic descriptions including vomiting of the esophageal epithelium in the form of a "tubular cast" [6]. Many patients are asymptomatic at the time of presentation and undergo EGD for other reasons. In our case, the patient presented with severe anemia and a positive fecal occult blood test. EGD was performed to determine the etiology of anemia and EDS was incidentally diagnosed.

Patients with EDS are older, more debilitated, immunosuppressed in some cases, and are more likely to be taking multiple medications $[3,7]$. In a recent case series by Hart et al, $73 \%$ of patients were taking a psychoactive agent at the time of diagnosis [1]. Our patient was not on medications at the time of diagnosis but had a social history of heavy alcohol consumption.

\section{Diagnostic strategy}

Diagnostic criteria for EDS have not been established. In a review of case series and case reports, EDS is proposed to 
be a clinical diagnosis supported by histological findings. Fulfillment of the three following endoscopic criteria is consistent with EDS: 1) strip(s) of sloughed esophageal mucosa $>2 \mathrm{~cm}$ in length; 2) normal underlying mucosa; and 3) lack of ulcerations or friability of immediately adjacent esophageal mucosa $[1,8,9]$. Histologically, the superficial portion of the squamous epithelium becomes detached and common findings include parakeratosis and intraepithelial splitting in the superficial layers of the epithelium. Furthermore, biopsies may not be necessary for all patients, particularly when EGD findings are classic for EDS and the patient is asymptomatic. When a coexisting diagnosis is suggested, biopsies may be necessary. Differential diagnoses include candidiasis, pill esophagitis, mucosal trauma, celiac disease, and others. In our patient, both the EGD and histologic findings supported the diagnosis of EDS, with concurrent findings of fungal hyphae on microscopy.

\section{Treatment}

EDS is a benign condition that has a classic endoscopic appearance. It has no specific treatment and the clinical course parallels the patient's underlying disease activity. Offending agents should be discontinued. Currently, there are no published surveillance guidelines [7, 10].

\section{Conclusion}

EDS is rare and is likely underestimated and underdiagnosed. It is a benign condition with an unknown etiology. EDS is associated with systemic diseases and commonly prescribed medications such as bisphosphonates and NSAIDS. However, this case for the first time highlights the possibility of fungal etiology as well. Upper endoscopy is important for the diagnosis. Histology is helpful to support the diagnosis. Therapy is supportive and involves stopping the offending agent and treating the underlying condition. Timely recognition and diagnosis of EDS may avoid unnecessary investigations and therapy.

\section{Funding}

This research received no specific grant from any funding agency in the public, commercial, or not-for-profit sectors.

\section{Conflicts of Interest}

The authors do not have any conflicts of interest or financial relationships to disclose.

\section{Author Contributions}

Conception and design, drafting, critical revision for important intellectual content, and final approval: Rashmee Patil, Mel Ona, Tagore Sunkara, Vinaya Gaduputi, and Madhavi Reddy.

\section{References}

1. Hart PA, Romano RC, Moreira RK, Ravi K, Sweetser S. Esophagitis Dissecans Superficialis: Clinical, Endoscopic, and Histologic Features. Dig Dis Sci. 2015;60(7):20492057.

2. Grin A, Streuker C. Esophagitis. Arch Pathol Lab Med. 2015;139:723-729.

3. Akhondi H. Sloughing esophagitis: a not so common entity. Int J Biomed Sci. 2014;10(4):282-286.

4. Brownschidle SS, Ganguly EK, Wilcox RL. Identification of esophagitis dissecans superficialis by endoscopy. Clin Gastroenterol Hepatol. 2014;12(9):e79-80.

5. Longman RS, Remotti H, Green PH. Esophagitis dissecans superficialis. Gastrointest Endosc. 2011;74(2):403-404.

6. Ma C, Limketkai BN, Montgomery EA. Recently highlighted non-neoplastic pathologic entities of the upper GI tract and their clinical significance. Gastrointest Endosc. 2014;80(6):960-969.

7. Purdy JK, Appelman HD, McKenna BJ. Sloughing esophagitis is associated with chronic debilitation and medications that injure the esophageal mucosa. Mod Pathol. 2012;25(5):767-775.

8. Carmack SW, Vemulapalli R, Spechler SJ, Genta RM. Esophagitis dissecans superficialis ("sloughing esophagitis"): a clinicopathologic study of 12 cases. Am J Surg Pathol. 2009;33(12):1789-1794.

9. Yogarajah M, Sivasambu B, Jaffe EA. Bullous systemic lupus erythematosus associated with esophagitis dissecans superficialis. Case Rep Rheumatol. 2015;2015:930683.

10. Hokama A, Yamamoto Y, Taira K, Nakamura M, Kobashigawa C, Nakamoto M, Hirata T, et al. Esophagitis dissecans superficialis and autoimmune bullous dermatoses: A review. World J Gastrointest Endosc. 2010;2(7):252-256. 